\title{
A CMOS-compatible Large-Scale Monolithic Integration of Heterogeneous Multi-Sensors on Flexible Silicon for IoT Applications
}

\author{
Joanna M. Nassar ${ }^{1}$, Galo A. Torres Sevilla ${ }^{1}$, Seneca J. Velling ${ }^{2}$, Marlon D. Cordero ${ }^{1}$, Muhammad Mustafa Hussain ${ }^{1 *}$ \\ ${ }^{1}$ Integrated Nanotechnology Lab and Integrated Disruptive Electronic Applications (IDEA) Lab, Computer Electrical Mathematical \\ Science and Engineering Division, King Abdullah University of Science and Technology (KAUST), Thuwal 23955-6900, Saudi \\ Arabia \\ ${ }^{2}$ Material \& Nanoscience Program, Faculty of Science, University of Waterloo, Waterloo ON, N2L-3G1, Canada \\ Corresponding Author's Phone: +966-544-700-072 and E-mail: muhammadmustafa.hussain@ kaust.edu.sa
}

\begin{abstract}
We report CMOS technology enabled fabrication and system level integration of flexible bulk silicon (100) based multi-sensors platform which can simultaneously sense pressure, temperature, strain and humidity under various physical deformations. We also show an advanced wearable version for body vital monitoring which can enable advanced healthcare for IoT applications.
\end{abstract}

\section{INTRODUCTION}

Flexible electronics are emerging class of electronics which can enhance the quality of life significantly. With its much success in advanced organic materials based display technology, it has significant potential in wearable technology too, especially for advanced healthcare and monitoring, further embracing Internet of Things (IoT) era. However, cointegration of silicon based data processing units and advanced materials based flexible sensors with simultaneous sensing capability calls for heterogeneous integration strategy. Especially challenging that widely used bulk monocrystalline silicon (100) is a rigid and brittle material. Scaling itself is not sufficient to co-integrate complex data processing ICs with large fan-outs on soft substrate with discrete components needed for a fully compliant wearable system which can nearly match human skin's Young's modulus and can follow the asymmetric terrain on its surface without skin irritation. Finally, from affordability and reliability perspective, developing a fully flexible bulk silicon (100) based multisensory platform can be a true game changer for IoT technology. In that regard, monolithic integration will be needed through heterogeneous on-chip integration of sensors and transistors for fully functional system.

Organic materials and elastomers have been commonly used to achieve flexibility in sensor development $[1,2]$. These polymeric materials are inherently flexible, yet offer limited electron mobility and exhibit a high threshold voltage for transistor operation leading to high power consumption [2]. The most advanced organic semiconducting materials offer mobilities on the order of $10 \mathrm{~cm}^{2} . \mathrm{V}^{-1} \cdot \mathrm{s}^{-1}$, e.g. Yuan et al. in 2014 showed mobilities of $43 \mathrm{~cm}^{2} \cdot \mathrm{V}^{-1} \cdot \mathrm{s}^{-1}$ using specially designed organic thin-film transistors (TFT), nevertheless two orders of magnitude below that of Silicon [2]. While they offer form-factor advantages, large power consumption, low mobility, and large subthreshold slope make their viability limited in fully functional practical wearable systems. While improvement upon these challenges has been a consistent research interest for scientific community, the only major challenge in conventional silicon based CMOS electronics is to make them flexible using a CMOS compatible technology, while preserving affordability and its robustness to sustain various physical deformation when worn.

By approaching flexibility from a CMOS perspective, we previously showed that silicon FinFETs could be made flexible using fully CMOS compatible processes while maintaining high-performance [4]. Since, silicon-based CMOS electronics are the most shovel ready candidates to meet the requirements for IoT application with lower power consumption, higher mobility, and less leakage than TFTs, their monolithic on-chip integration of active matrix sensors with high performance CMOS circuit components (viz. microprocessor and power management) is critical [5]. Therefore, in this paper we report large-scale monolithic integration of advanced heterogeneous multi-sensors platform using CMOS compatible flexible bulk silicon (100) based CMOS sensors and electronics. The fabricated active-matrix sensor array can sense pressure, temperature, strain and humidity simultaneously. We also present the opportunity for an advanced wearable version for body vital monitoring under physical deformations.

\section{INTEgRATION Process AND METhOdOLOGY}

The monolithic integration of sensor networks on a flexible $\mathrm{Si}$ platform is successfully completed through a CMOS compatible approach (Fig. 1). Multisensory capabilities including temperature, strain, humidity, and pressure were demonstrated using a variety of designs and architectures. Temperature sensors design employed standard resistive temperature detection (RTD), strain sensors incorporated dualaxis gauge structures, humidity sensing used interdigitated electrodes beneath a polyimide (PI) sensing film, and pressure sensing relied on parallel plate capacitance with a highly compressive polydimethylsiloxane (PDMS) dielectric barrier [6]. 
The heterogeneous integration process is illustrated in the schematic of Fig. 2, and begins with an oxidized bulk Si (100) wafer with a $300 \mathrm{~nm}$ thermal $\mathrm{SiO}_{2}$ layer. Then $10 \mathrm{~nm} / 200 \mathrm{~nm}$ of Ti/Au was sputtered and patterned using Ar plasma to form the first metal layer. Then, $1 \mu \mathrm{m}$ polyimide (PI) is spin coated, cured at $350^{\circ} \mathrm{C}$, and patterned using oxygen $\left(\mathrm{O}_{2}\right)$ plasma and tetrafluoromethane $\left(\mathrm{CF}_{4}\right)$ in a reactive ion etching (RIE) tool. PI etching was ensured using a $200 \mathrm{~nm}$ aluminum (Al) hard mask layer to form the humidity sensing layer. Afterwards, the PI layer was surface treated with $\mathrm{O}_{2}$ plasma to improve its adhesion with the second metal layer to be deposited. Finally, the pressure sensor is completed by spin coating and curing 200 $\mu \mathrm{m}$ of polydimethylsiloxane (PDMS) as the pressure sensing material. The PDMS deposition is performed last due to high aspect ratios that would otherwise cause deposition nonuniformities, introducing challenges to alignment and lithography. RIE patterning of PDMS was performed through a $4 \mu \mathrm{m}$ Copper $(\mathrm{Cu})$ electroplated hard mask. The gases used are composed of a 10:1 ratio of SF6:O2, which are not selective to photoresist but highly selective to $\mathrm{Cu}$. Excess $\mathrm{Cu}$ is then removed using a wet etchant and finally, the platform is flexed down to $20 \mu \mathrm{m}$ thickness using the aforementioned soft back etch technique we have developed [4].

\section{RESULTS AND DISCUSSION}

In this work, we focus on assessing the performance of pressure, temperature, strain, and humidity sensors when exposed to external stimuli, as well as the bending effect to monitor their robustness for wearable IoT devices.

\section{A. Sensor Characterization and Response to Stimuli}

Tensile bending of the $5 \mathrm{~cm} \times 3 \mathrm{~cm}$ sample to a radius of $0.5 \mathrm{~cm}$ does not induce substantial strain $(<0.03 \%)$ on films (Fig. 3). While strain in the bending direction exhibits a linear behavior (Fig. 4 ) with gauge factor $\approx 0.69$, the strain in the nonbending direction fluctuates little $( \pm 14.6 \%)$ about the $334.82 \Omega$ mean, indicating material resilience under tight bending radii, making flexible Si viable for interconnects.

Temperature sensing was characterized before and after flexing process, and under bending conditions (Fig. 5). Consistent linear behavior (correlation coefficient $\mathrm{R}=0.989 \pm$ 0.015) was observed, with minor fluctuations of the temperature coefficient of resistance $\left(\mathrm{TCR}=1.6 \times 10^{-3} /{ }^{\circ} \mathrm{C} \pm\right.$ $\left.1 \times 10^{-4} /{ }^{\circ} \mathrm{C}\right)$, and a stress-induced upward shift $(\approx 3 \Omega)$ is observed due to bending. Pressure sensing tested under bending conditions displayed a linear regime beginning at $\approx 37 \mathrm{~Pa}$ with a sensitivity to human touch of $\approx 2 \mathrm{kPa}$ at $2 \mathrm{~cm}$ bending radius (Fig. 6). We observe a shift upwards in the capacitance value with a decrease in the bending radius as dielectric thickness decreases. Nevertheless, the overall shape and behavior of the plot remains the same, with a slight increase in sensitivity with reduced bending radius due to a decrease in the contact area between fixed-masses $\left(\mathrm{S}=0.012 \mathrm{kPa}^{-1}-0.023 \mathrm{kPa}^{-1}\right)$ (Fig. 6). As for the humidity sensor, hydroxyl-group sensitive polyimide-based sensor demonstrated response to variation in environmental humidity and complete immersion in ethanol and water, through a change in measured dielectric permittivity (Fig. 7). Fig. 7 displays the sensor's behavior under a concave bending radius $\mathrm{R}=2 \mathrm{~cm}$, demonstrating measurable difference between detected relative humidity levels. Relative to room humidity, skin hydration level displays a negative value of $\Delta \mathrm{C} / \mathrm{C}_{0}$, meaning the skin has a lower level of moisture than the surrounding environment. Similarly, a $1 \mathrm{ml}$ water droplet on the sensor leads to an increase in the signal, highlighting the saturation level with maximum moisture. In contrast, when a 1 $\mathrm{ml}$ ethanol droplet is in contact with the sensor, relative humidity levels drop with respect to pure water, since the solution contains less hydroxyl ions to react with the PI film.

\section{B. Application in Body Vitals Monitoring}

Our electronic skin displays viable sensing characteristics under bending conditions and offers potential in health monitoring applications: blood pressure, heart-rate, temperature, and sweat. Epidermal blood pressure collected from the radial artery using a pressure sensor has a dampened signal $(\approx 70 \%)$ [6]. Additionally, our strain sensor allows $\mu \mathrm{m} / \mathrm{mm}$ scale detection of epidermal irregularities (inducing $\approx$ $1.3 \%$ strain), corresponding to $\approx 6000 \%$ increase in resistance. In combination with temperature and sweat detection, this technology highlights the efficacy of symptom monitoring in patients. Furthermore, for any developed wearable system, packaging remains a crucial part to be addressed. Here we also show a low-cost technique to conformally package flexible Si based electronics in flexible 3D printed casing as decal electronics (Fig. 8). This affordable technique protects the devices while retaining mechanical flexibility and robustness.

\section{CONCLUSION}

Flexible version of bulk monocrystalline silicon (100) can present significant advantages and a critical step in monolithic heterogeneous on-chip integration needed for IoT devices specially focusing on advanced healthcare. Therefore, we have reported state-of-the-art CMOS compatible large-scale integration of heterogeneous multi-sensory array on a flexible bulk silicon (100) substrate which can simultaneously sense pressure, temperature, strain and humidity under various physical deformation. We also show an advance version of the device in a 3D printed packaged wearable gadget format (Fig. 8) which enables a pragmatic route to realize IoT devices focusing on integration of human-machine interface for wellness and healthcare technology.

\section{REFERENCES}

[1] T. Someya, et al., "Conformable, flexible, large-area networks of pressure and thermal sensors with organic transistor active matrixes," Proc. Natl. Acad. Sci. U. S. A., vol. 102, no. 35, pp. 12321-12325, Mar. 2005.

[2] D. J. Lipomi, et al., "Skin-like pressure and strain sensors based on transparent elastic films of carbon nanotubes," Nat. Nanotechnol., vol. 6 , no. 12, pp. 788-792, Oct. 2011.

[3] Y. Yuan, et al., "Ultra-high mobility transparent organic thin film transistors grown by an off-centre spin-coating method," Nat. Commun., vol. 5, no. 3005, Jan. 2014.

[4] G. A. Torres, M. T. Ghoneim, F. Hossain, J. P. Rojas, A. M. Hussain, and M. M. Hussain, "Flexible nanoscale high-performance FinFETs," ACS Nano, vol. 8, no. 10, pp. 9850-9856, Sep. 2014.

[5] J. P. Rojas, et al., "Transformational silicon electronics," ACS Nano, vol. 8, no. 2, pp. 1468-1474, Jan. 2014.

[6] M. Ward and J. A. Langton, "Blood pressure measurement," Continuing Education in Anaesthesia, Critical Care \& Pain, vol. 7, no. 4, pp. 122126, 2007. 

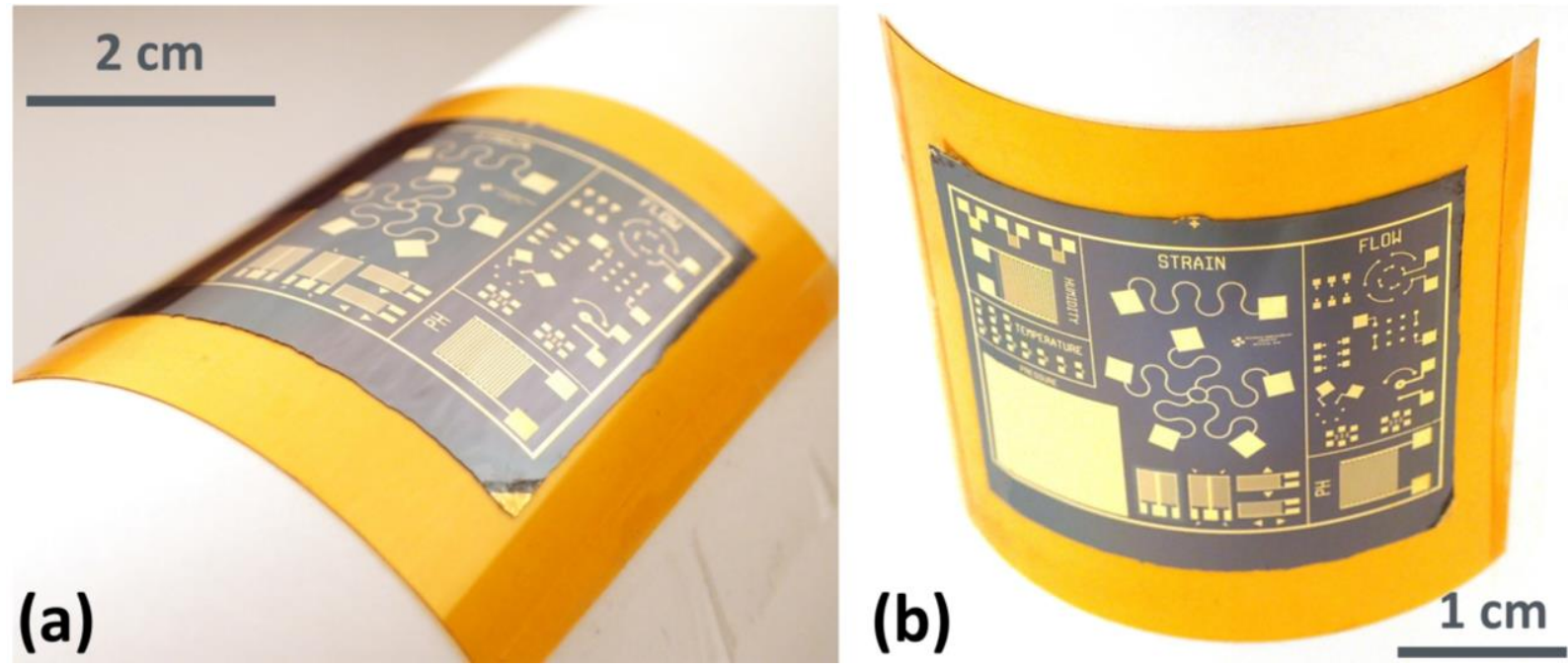

Figure 1. Digital photographs of flexible and bendable Si-based electronic skin with multisensing capabilities.
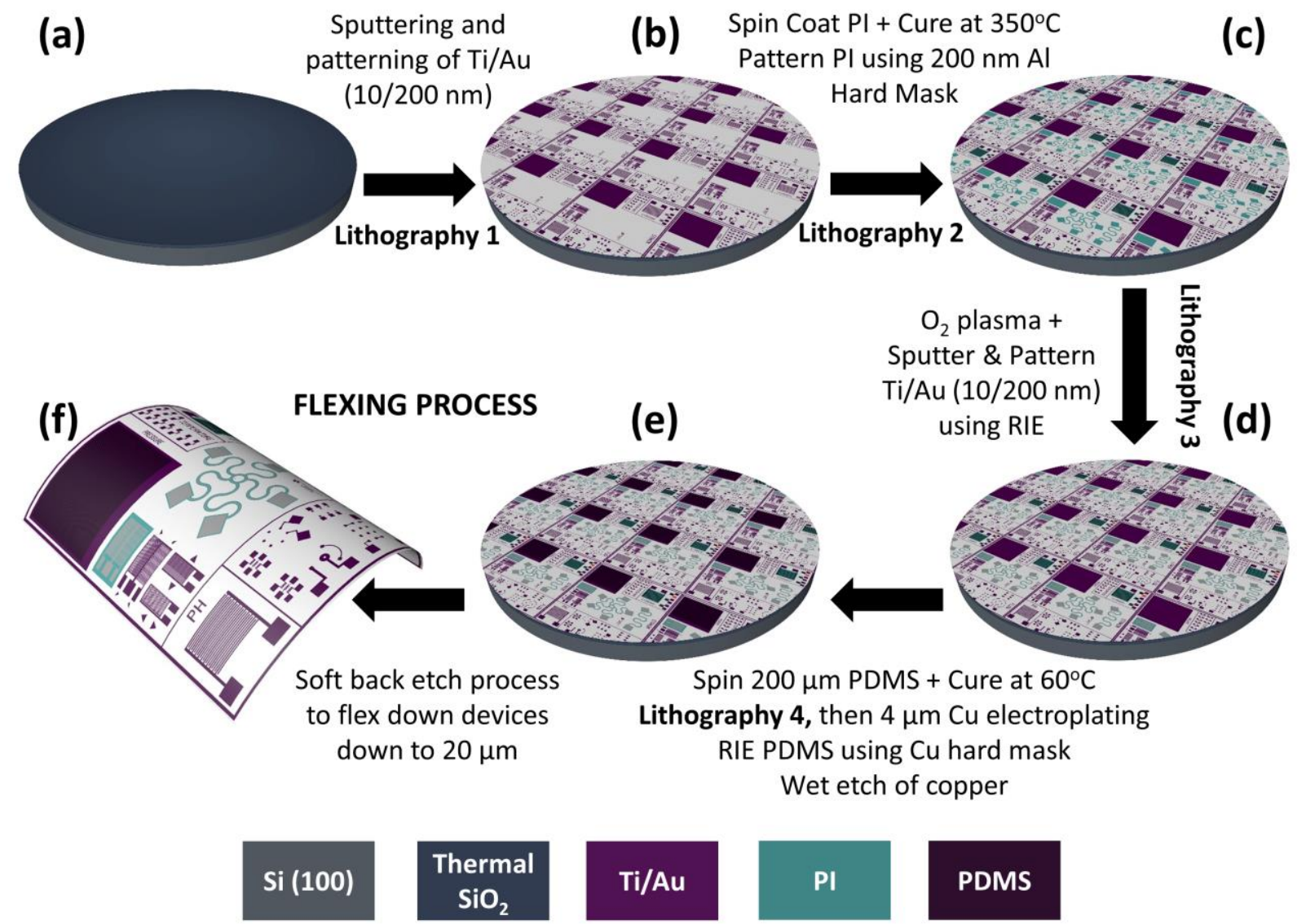


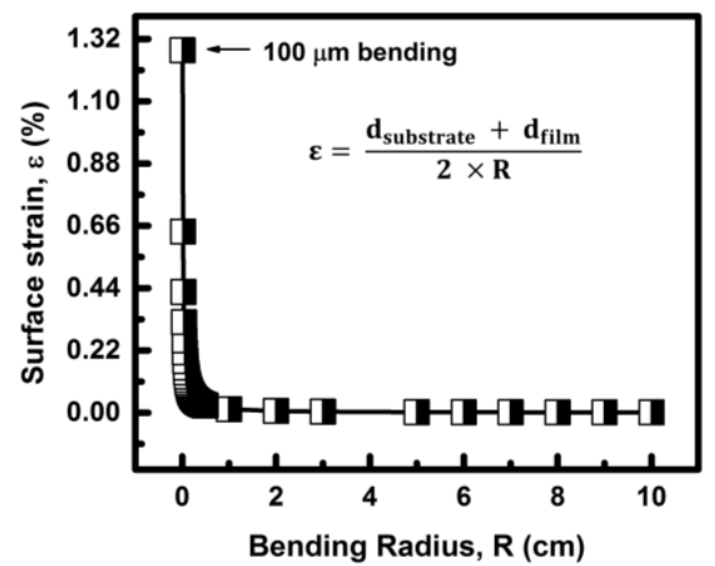

Figure 3. Bending-induced strain percentage with respect to different bending radii, calculated for a flexible silicon substrate of $20 \mu \mathrm{m}$ thickness.

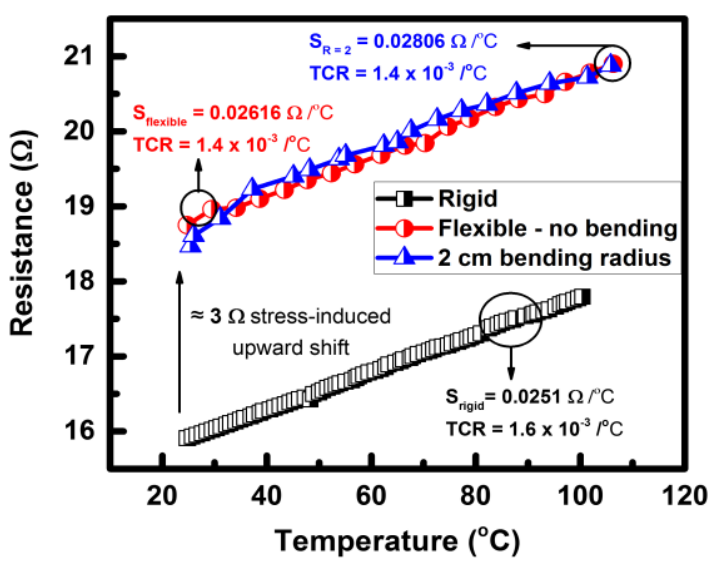

Figure 5. Temperature sensitivity before flexible, after flexing, and under tensile bending condition with $\mathrm{R}=2 \mathrm{~cm}$.

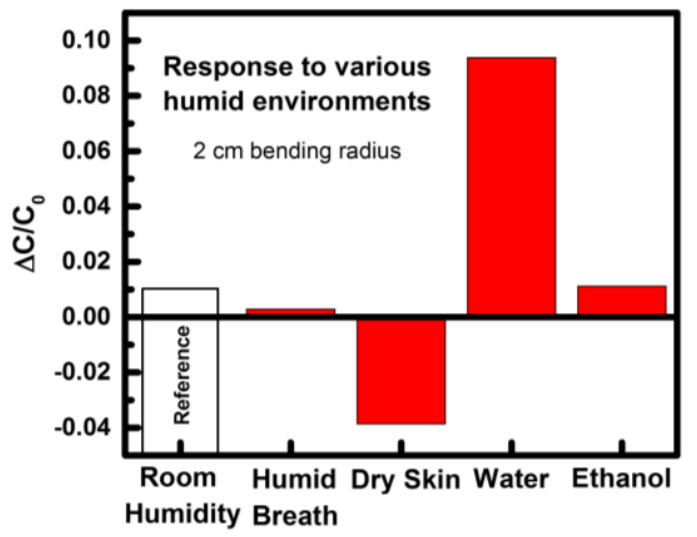

Figure 7. Differential humidity response to different relative humidity levels corresponding to humid breath, dehydrated skin, water detection, and ethanol on the surface of the sensor.

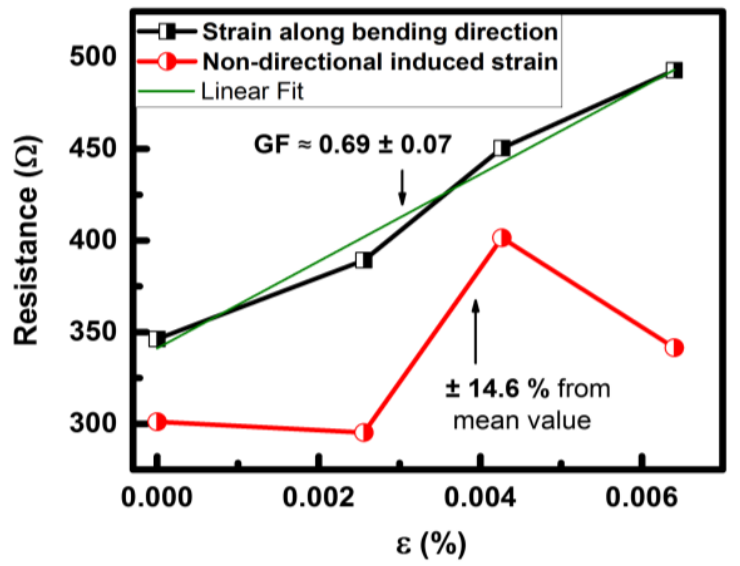

Figure 4. Resistance changes during biaxial strain sensing. Strain along bending direction underlines a linear increase in resistance, whereas the non-directional strain displays small fluctuations.

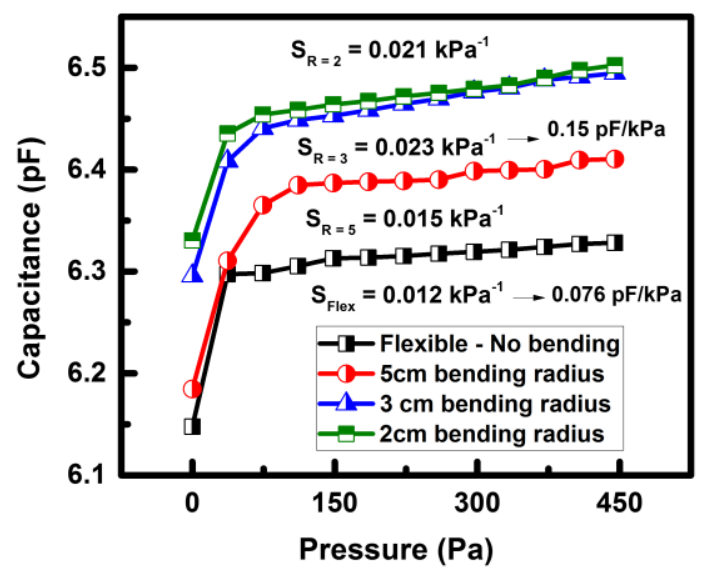

Figure 6. Pressure sensitivity plots before bending and under various tensile bending condition down to a minimum $\mathrm{R}=2 \mathrm{~cm}$.

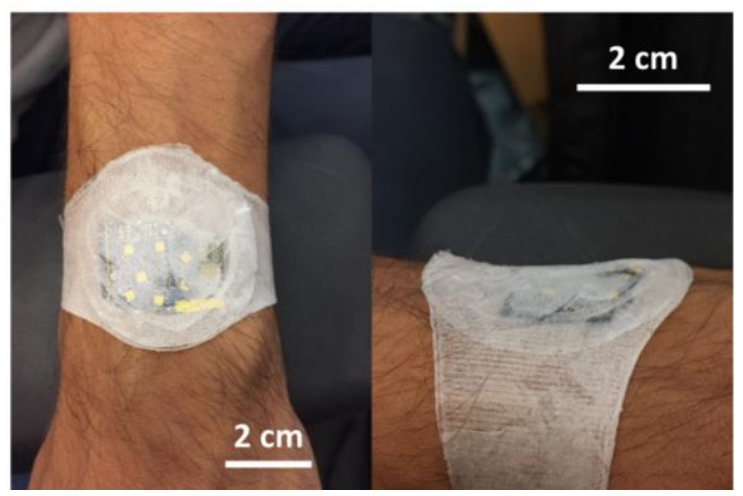

Figure 8. Flexible electronic skin embedded in a low-cost flexible 3D printed package, designed for conformal wear around the wrist in IoT monitoring devices. 